[Vicino Oriente XVI (2012), pp. 29-39]

\title{
CATTIVI PRESAGI: ANALISI DELLA RAFFIGURAZIONE DELLA STANZA 132 DEL PALAZZO REALE DI MARI
}

\author{
Paolo Sferrazza - Sapienza Università di Roma
}

\begin{abstract}
Mari is an ancient site located in odiern Syria in the modern village called Tell Hariri; since the beginning of the excavations was clear to archaeologists the importance of the site and especially of the Royal Palace. Inside this building researchers have found many pieces of different wall paintings located mainly in Court 106 and in Hall 132. The last is the subject of this article, particularly the register who shows the god Sin seated in a throne carved in the mountain. To Parrot, Margueron and Matthiae, the principal researchers who studied and debated this wall painting, this register seems somewhat enigmatic. It becomes more clear, in my opinion, if we consider it a visual representation of an ancient ritual called "substitute king"; in fact if we do so each character, and especially every iconographic oddity (for example god Sin seated in a throne in a mountain in which usually sits Shamash, mace symbol of royalty in the hand of a "high priest" and so on), seems to be in the right place with the right meaning. Unfortunately, if this analysis is correct, this is the only know representation of "substitute king" rite and we can't do comparison with other depictions that could strengthen this hypothesis.
\end{abstract}

Keywords: Mari; pittura; “sostituzione del re”; Stanza 132; Palazzo Reale

\section{INTRODUZIONE}

Questo contributo vuole, per mezzo di una ipotesi, gettare luce sulla raffigurazione parietale nella Stanza 132 (fig. 1) del Palazzo Reale di Zimri-Lim di Mari. In particolare si cercherà di chiarire il significato del secondo registro maggiore in basso, la scena con il dio Sin, associandola al rito di sostituzione del re. Purtroppo, ad oggi non vi è nessun precedente che attesti una rappresentazione figurata di questo rito. Conseguentemente non è stato possibile corroborare questa tesi con paralleli iconografico-artistici, nonostante, come si vedrà più avanti, l’ipotesi abbia senza dubbio il pregio di restituire un senso logico alla raffigurazione, connotando precisamente ogni figura ad una funzione coerente ${ }^{1}$. Tre sono gli autori che principalmente hanno studiato e discusso la rappresentazione pittorica in questione: A. Parrot nel 1958², seguito dalle conclusioni di J.C. Margueron ${ }^{3}$ ed infine di P. Matthiae $^{4}$. Le ultime due differiscono in moltissimi punti dalla prima, mentre coincidono in

1 In un dipinto che altrimenti è stato definito atipico da parecchi studiosi tra i quali lo stesso Margueron (2004, 419).

2 Parrot 1958a, 70-73; 1958b, 63-66.

3 In particolare, l'archeologo francese si è preoccupato di analizzare lo sviluppo diacronico del Palazzo Reale secondo uno studio funzionale e architettonico degli ambienti di comunicazione, al fine di datare in maniera più sicura il palazzo e i suoi dipinti (Margueron 1982, 331-332, 417-419).

4 Matthiae 2000, 94-96. Anche A. Moortgart ha studiato dal punto stilistico e iconografico l'opera in questione, collocandola al periodo di Gudea di Lagash. Se l'analisi stilistica di Moortgart è precisa e fluida, allo stesso tempo non è possibile ignorare i dati del contesto archeologico. Secondo Margueron, che pur ammette le influenze stilistiche arcaicizzanti, quel settore del palazzo venne costruito da Hanun-Dagan nel 2000 a.C. circa. Appare quindi improbabile che quel settore del palazzo fosse eretto già nel XXII secolo, nonostante la pittura mostri evidenti richiami neo-sumerici ciò infatti non vuol dire che sia stata realizzata in quel secolo. Al contrario è risaputa la tendenza del potere politico nel Vicino Oriente a rifarsi ad antichi canoni ritenuti 
molti punti tra loro ${ }^{5}$ In particolare Matthiae ${ }^{6}$ sostiene una datazione ${ }^{7}$ leggermente più tarda di Margueron per le pitture della Stanza 132.

\section{IL RITO DI SOSTITUZIONE DEL RE}

Le notizie più ampie riguardo il rito di sostituzione del re vengono dalle lettere assire inviate ai re Esarhaddon (681-669 a.C.) e Assurbanipal (668-631 a.C.), dove il rituale viene menzionato più volte ${ }^{8}$; da questa corrispondenza si evince che il rituale seguiva un percorso molto articolato:

- intronizzazione ufficiale del sostituto del re, quest'ultimo è presentato al suo popolo;

- il rito di intronizzazione è una consacrazione ${ }^{9}$ del nuovo re, inoltre l'uso delle vere insegne della regalità lo pone a contatto col mondo divino ${ }^{10}$;

- il re sostituto deve recitare dinanzi al dio Shamash i cattivi presagi che sono causa di pericolo per il regno e che quindi comportano la necessità del sacrificio ${ }^{11}$. La recitazione avveniva per mezzo dell'evocazione di Shamash come massimo giudice celeste;

- dopo un periodo di regno piuttosto variabile il sostituto viene ucciso.

Il rito di sostituzione del re è un rito sacrificale cruento in cui la vittima ha funzione di capro espiatorio $^{12}$, e nasce dalla credenza che il male possa trasferirsi su un altro essere (sia umano che animale) o su un feticcio costruito ad hoc ${ }^{13}$ : essenziale per questo passaggio era comunque una somiglianza o contatto tra le due persone coinvolte nel rito, in questo caso il sostituto diviene a tutti gli effetti un re mutandosi in vittima del presagio e delle correnti negative ${ }^{14}$. Questo rituale estremo ${ }^{15}$, che prevede un sacrificio umano, non deve

formanti la regalità stessa (Gates 1984, 77; Margueron 2004, 416).

5 Margueron, nonostante dati con una certa precisione la costruzione dell'area di cui qui si scrive, accoglie l’ipotesi di datazione di Matthiae come possibile (Margueron 2004, 416).

6 Per Matthiae la raffigurazione sarebbe da collocare poco prima della presa della città da parte di ShamshiAddu (Matthiae 2000, 95).

7 Per questa analisi si è deciso di utilizzare, norma adottata anche dagli studiosi qui menzionati, la datazione cosiddetta "media".

8 Parpola 2007, XXII-XXXII.

9 Sono Hubert e Mauss che per la prima volta si oppongono in modo efficace alle teorie di Taylor riguardo $\mathrm{i}$ sacrifici. Quest'ultimo infatti relegava il sacrificio alle sole culture totemiche, cosa efficacemente smentita dallo studio e dai dati riportati da Hubert e Mauss (Hubert - Mauss 1981, 6).

10 La sacralizzazione della vittima è uno dei passi imprescindibili dei sacrifici cruenti e non. Senza questo momento il rituale non avrebbe senso, specie in riferimento al rito di sostituzione del re dove, il momento della sacralizzazione, corrisponde all'atto di consacrare e intronizzare il nuovo re "fantoccio" (Hubert - Mauss 1981, 9-19).

11 Parpola 2007, lettere: 26 e 30.

12 Frankfort 1978, 262.

13 Il concetto di male come un fardello che può passare da un individuo ad un altro è stato magnificamente ripreso in età greca con Eschilo e le sue opere, dove il male compiuto dagli avi si trasmetteva di generazione in generazione all'interno del nucleo famigliare sino all'espiazione finale. In questo caso assistiamo ad una sopravvivenza di una credenza antica in epoche successive.

14 Brelich 1991, 37.

15 Hubert e Mauss distinguono il dono dal sacrificio, dicendo che il secondo ha una potenza rituale maggiore ed è usato in casi considerati più estremi. Ciò sarebbe dimostrato dal fatto che nel primo caso, una parte del dono, 
impressionare o apparire irreale ai nostri occhi, poiché non bisogna mai dimenticare che la definizione di individuo, vita e morte era diversa rispetto a quella che oggi abbiamo. Vi era un detto antico, circa del XVIII secolo a.C., la cui prima attestazione è forse nel regno di Mari, che diceva: "che io possa andare in tua vece (contro il pericolo)"16; questo è un prezioso indizio che permette di intuire quanto fosse naturale per quella società accettare un simile mezzo per la salvaguardia del re. Purtroppo definire questo rituale è difficile a causa della frammentarietà delle lettere pervenuteci. Nello specifico è complesso sapere se vi si ricorresse spesso oppure solo in casi estremi, cioè se ogni eclissi avvistata comportasse o meno l'attuazione di questo rituale o quante volte in media. Infatti, se da un lato le attestazioni di questo rituale risultano piuttosto scarse, dall'altro solo nel regno di Esarhaddon, secondo i calcoli di S. Parpola, si dovette applicare la sostituzione del re quattro volte ${ }^{17}$.

Le lettere assire, facenti parte della corrispondenza di Esarhaddon e Assurbanipal che attestano la pratica di questo rituale, sono in totale $\operatorname{trenta}^{18}$. Sino ad oggi le corrispondenze ritrovate ci dicono che questo rito si svolgeva in occasioni delle eclissi ${ }^{19}$ di Sole, Luna ${ }^{20}$, Mercurio, Venere, Giove e Saturno ${ }^{21}$; ciò non era sistematico, ma avveniva solo quando gli indovini credevano fosse necessario: a riprova di quanto detto vi è l'esistenza di molte eccezioni dovute a diversi fattori astrologici ${ }^{22}$. L'osservazione del disco lunare o solare era importante, perché stabiliva quale parte del paese veniva coinvolta nel caso di un'eclissi parziale $^{23}$. Nel caso in cui l'eclissi era totale o anulare erano coinvolti tutti i regnanti nel cui regno il fenomeno veniva osservato.

Oltre i documenti del periodo neo-assiro, ve ne sono altri di epoche ben più anteriori che dimostrano l'esistenza e l'uso di questo rituale durante un arco cronologico vastissimo: è il caso dei re Erra-Imitti (1868-1861 a.C.) ed Enlil-Bani (1860-1837 a.C.), due re della

oltre che dalla divinità, viene consumata dalla comunità; nel secondo caso è invece prevista la distruzione totale della vittima sacrificale e la sua immolazione alla divinità (Hubert - Mauss 1981, 9-19).

16 Frankfort 1978, 263.

17 Parpola 2007, XXII-XXXII.

18 Parpola 2007, lettere: 25-28, 30-32, 77, 134-139, 166-167, 179, 185, 205, 232, 235-236, 249, 257, 278-280, 292, 298-299, 317, 344.

19 Come mostrato dai testi, a Mari le osservazioni delle eclissi erano sistematiche. Alle osservazioni erano anche associati elenchi di azioni e significati (Durand 1995, 376-377).

20 A Mari è stato ritrovato un resoconto astrologico che descrive un'osservazione lunare. In questo caso il divinatore, Asqudum, non è soddisfatto del risultato poco chiaro ed opera l'aruspicina per avere maggiori certezze. Secondo Koch-Westenholz e Koch questa sarebbe una prova della scarsa considerazione di cui godeva l'astrologia a Mari. Ma, come sostiene lo stesso Durand, i profeti e gli indovini a Mari sono considerati dei messaggeri così le loro predizioni sono sempre poste al vaglio di una prova, che spesso è l'epatoscopia. Quindi il fatto che un fenomeno astrologico osservato venga vagliato con un altro sistema divinatorio è assolutamente normale nel sistema divinatorio adottato a Mari (Durand 2000, 91-93; Durand 1995, 318, 376; Koch-Westenholz - Koch 1995, 37).

21 Parpola 2007, lettera: 3.

22 Ne è un esempio la tavola XX dell'enuma anu enlil, dove si raccomanda che se durante un'eclissi di luna Giove è visibile nel cielo, allora il re è salvo ma rischia la vita un alto dignitario della corte.

23 In questo sistema il sole e la luna venivano divisi in quattro settori. Se era coinvolta la parte superiore degli astri erano in pericolo i re dell'ovest (Amurru), la parte inferiore interessava i re assiri (Subartu), quella destra Babilonia ed Akkad, quella sinistra l'Elam (Parpola 2007, lettera 3). 
dinastia di Isin. I resoconti trovati ci dicono che il re Erra-Imitti pone il giardiniere EnlilBani nel trono come re sostituto ${ }^{24}$, ma durante il regno fantoccio di Enlil-Bani, Erra-Imitti muore assaggiando un brodo bollente; Enlil-Bani riuscirà a farsi confermare $\mathrm{re}^{25}$ dopo la morte del vero sovrano, mantenendo il potere per svariati anni.

Il sostituto veniva solitamente scelto tra i prigionieri di guerra, criminali, nemici politici o cittadini di bassa estrazione sociale; si tentava, insomma, di scegliere una persona la cui morte e sacrificio sarebbero state "accettabili"”2. Il sostituto indossa tutti i simboli della regalità: mantello, tiara, le armi, lo scettro ${ }^{27}$; non solo, ma, come detto sopra, anche materialmente il sostituto diviene il re ufficiale, mentre quello che prima era tale si rifugia con parte della corte evitando assolutamente qualsiasi contatto con il popolo, il quale, probabilmente, non era neanche a conoscenza di quanto avvenisse in realtà̀ ${ }^{28}$.

In questo periodo di regno, che aveva un tempo massimo di 100 giorni ma che più spesso poteva durare molto meno ${ }^{29}$, il sostituto godeva di tutti gli agi dovuti ad un sovrano, ma sicuramente non aveva alcun diritto decisionale né alcun potere effettivo, dovendo solo interpretare il ruolo del re ${ }^{30}$. Inoltre, come sostenuto dallo stesso Parpola ${ }^{31}$, pare che il re avesse con sé circa cento guardie armate con lo scopo non tanto di difenderlo, quanto di evitare un tentativo di fuga. Terminato il tempo previsto per il regno fittizio il re, probabilmente consapevole della sua fine $e^{32}$, veniva sacrificato. Durante questa fase il male preannunciato dai cattivi presagi viene canalizzato e mandato lontano insieme allo spirito del re sostituto; anche il funerale doveva seguire pedissequamente la trafila prevista per la morte di un re, così il cadavere veniva preparato ed esposto al pubblico in pompa magna e il corpo veniva probabilmente tumulato con tutti gli onori previsti in un mausoleo costruito apposta $^{33}$. Facevano eccezione dei riti aggiuntivi di esorcismo eseguiti con lo scopo di

24 Bottéro 1991, 155.

25 La narrazione di questo evento dimostra come, a prescindere dall'estrazione sociale del sostituto e dal motivo rituale per cui questo rito era effettuato, l'efficacia rituale e materiale del rituale era reale. Ciò permette al re fantoccio, alla morte del re vero, di intronizzarsi.

26 Bottéro 1991, 155.

27 Frankfort 1978, 263.

28 Non si ha prova né della consapevolezza del popolo né del contrario riguardo questo rito; nonostante ciò questo dubbio è di poco valore, dato che il rito non avrebbe perso la sua efficacia in nessuno dei due casi. Il potere di questo sacrificio è insito nel suo apparato simbolico, e su quanto un simbolo avesse potere presso una società in cui tutto è materializzazione della volontà divina.

29 La durata del regno del re sostituto variava a seconda di eventuali altri presagi negativi che andavano accorpati all'evento scatenante il rito. Infatti se pochi giorni dopo l'eclissi avveniva qualcos'altro interpretabile come un segno negativo, o si sapeva dovesse avvenire (il caso di un'altra eclissi il mese successivo ad esempio), il sostituto continuava a regnare accumulando presagi negativi da rigettare tutti insieme nel momento dell'uccisione. In questo modo il regno poteva durare dalla settimana, al mese o al massimo 100 giorni (Parpola 2007, XXV).

30 Bottéro 1991, 158.

31 Parpola 2007, XXV.

32 Il re sostituto recitava i presagi scritti dagli astrologi dinanzi la statua del dio Shamash (Parpola 2007, lettere: $26,30,279)$.

33 Queste notizie si trovano in un resoconto di un certo Mar-Istar, rappresentante del re Esarhaddon a Babilonia. Questo documento è importante in quanto apre una piccola finestra sull'uso anche politico di questo rito; infatti Mar-Istar scrive, in questo resoconto, che il prescelto sostituto è il figlio di un esponente della corte 
scacciare con fermezza le forze negative imbrigliate alle figure dei sacrificati ${ }^{34}$. Ogni sacrificio prevede una serie di operazioni purificatorie cumulabili, spesso in grande numero, che operano al fine di spezzare e spazzare ogni legame malvagio rimasto dopo la conclusione del rito. In questo caso subito dopo la sepoltura dei due cadaveri, si bruciavano tutte le insegne regali per eliminare ogni corrispondenza del sostituto col ruolo di re una volta finito il suo compito. Poi si passava ad esorcismi praticati direttamente sul re ed infine alla "pulizia" del Palazzo Reale, che si articolava in svariati passaggi e si concentrava nei luoghi di rappresentanza del palazzo: gli ingressi, la corte e la sala del trono ${ }^{35}$. Terminato il decorso rituale previsto, il re poteva ufficialmente tornare al suo ruolo nel palazzo.

\section{LA STANZA 132}

La Stanza 132 del palazzo di Zimri-Lim è stata tema di dibattito, sia per quanto riguarda la sua funzione, sia per quanto concerne i suoi dipinti. Nel primo caso, Parrot sostiene che essa abbia funzione di sala d'udienza, motivando ciò grazie alla presenza di diversi elementi: una scala semi-circolare a tre gradini che dava accesso, dalla Corte 131, alla Stanza 132 una coppia di nicchie che dovevano trovarsi nella parete sud, ma che vennero successivamente chiuse dopo una ricostruzione dovuta ad un incendio ${ }^{36}$; un podio posto al centro della parete sud della Stanza, in asse con l'ingresso alla Stanza; delle decorazioni rilevanti sulla parete ovest. Secondo la ricostruzione di Parrot, il podio doveva essere il punto dove era sistemato il trono del re, che era a sua volta circondato da pitture in cui erano presenti scene mitiche e cultuali ${ }^{37}$. Infine la Corte 131 , che per la sua grandezza poteva ospitare molte persone, fungeva da anticamera per la sala d'udienza.

Margueron, procedendo negli studi e negli scavi, ha un'idea diversa circa la funzione della Stanza 132. Egli, infatti, sostiene che non vi sia alcuna menzione riguardo ad una sala d'udienza nelle tavolette ritrovate ${ }^{38}$, oltre al fatto che non vi è alcun esempio di un utilizzo, nei palazzi vicino orientali, di una sala d'udienza quando è già presente una sala del trono ${ }^{39}$. Margueron quindi ritiene trattarsi di una cappella, sia per la cura data all'ingresso della stanza, che per le scene religiose dipinte ed infine paragona la morfologia della Stanza 132, e il suo sistema di relazioni col palazzo, alla cappella del tempio di Ishtar Kititum a Ischali $^{40}$. Le raffigurazioni dipinte trovate nella Stanza vengono attribuite da Parrot, come del resto tutto l'edificio palatino del II millennio, all'epoca di Zimri-Lim di Mari ${ }^{41}$. La composizione (fig. 1) doveva trovarsi nella parete occidentale della Stanza all'altezza di due metri dal suolo; originariamente è stata ritrovata in pessimo stato, ridotta in molti

babilonese. Forse questa scelta era dettata dalla volontà di reprimere i malcontenti dovuti al dominio assiro (Bottéro 1991, 160-161; Parpola 2007, lettera 207).

34 Bottéro 1991, 162.

35 Bottéro 1991, 262-263.

36 Parrot 1958b, 63.

37 Queste avevano lo scopo di legittimare, e allo stesso tempo di mostrare, il potere del re e la connessione del suo mandato terreno con il divino (Parrot 1958b, 65).

38 Margueron 1982, 331.

39 Margueron 1982, 332.

40 Margueron 1982, 332.

41 Parrot 1974, 180. 
frammenti al suolo, oggi dopo una ricostruzione ed un restauro mostra 5 registri $^{42}$. Il soggetto di questo studio è il quarto registro partendo dall'alto, esso mostra una scena sacrificale ${ }^{43}$ complessa con diverse figure disposte non casualmente. Partendo da sinistra si osservano immediatamente un toro "passante" ${ }^{44}$, come ben si nota dalla zampa che si protende in avanti, rappresentato su di una montagna. Sempre sul monte, assiso su un trono ricavato dalla roccia, vi è stranamente ${ }^{45}$ il dio-Luna Sin facilmente riconoscibile per la tiara sormontata da una falce lunare. Sin indossa una veste bianca e porta una lunga barba nera, protende una mano in avanti in segno di accoglienza o benedizione. Di fronte a Sin vi è un piccolo altare bianco, o un podio, e sopra di esso un vaso con quelle che sembrano delle fiamme ${ }^{46}$ o, meglio, fumi d'incenso; davanti al podio vi sono due vasi che raccolgono i liquidi versati durante un'offerta effettuata da un secondo personaggio. Questo secondo personaggio è stato spesso definito come la rappresentazione di un re ${ }^{47}$; in effetti, a favore di questa interpretazione, si possono far notare alcune particolarità iconografiche tipiche come la tiara tonda bianca ${ }^{48}$ ed il mantello a balze di lana; questo ipotetico re effettua un gesto del tutto particolare con la mano libera, la destra, che si trova adesa al fianco opposto quasi dovesse stringere qualcosa o evidenziare un'assenza, una mancanza. Del resto, una figura che offre una libagione ad una divinità, solitamente, leva la mano libera, col palmo rivolto verso il proprio viso, alla divinità49. Questo personaggio, l'unico oltre Sin ed il toro a trovarsi sul monte, è inoltre di altezza inferiore rispetto alle due figure che gli stanno dietro. Quest'ultimo aspetto è abbastanza insolito dato che il re è sempre rappresentato di altezza superiore rispetto a chiunque tranne che al dio. Alle spalle del re si trovano altre due figure: una è la tipica dea intercedente ${ }^{50}$ che indossa una tiara con due corna, rappresentata frontalmente ${ }^{51}$, indossa un mantello a balze di lana ed ha le mani levate in un, iconograficamente noto, atteggiamento di protezione. Alle sue spalle vi è, infine, la figura di un sacerdote ${ }^{52}$ rappresentato nell'atto di reggere e protendere in avanti un vaso a collo

42 Margueron 2004, 418.

43 Parrot 1958a, 76.

44 Parrot scrive che potrebbe trattarsi del dio Dagan, in questo caso associato ad un'idea di fertilità (Parrot 1958a, 81)

45 Come notato sia da Matthiae e da Margueron, la presenza del dio Sin assiso nella montagna, dove normalmente dovrebbe trovarsi un dio solare come Shamash, è una deviazione dallo standard iconografico che vede sempre Shamash sorgere da un monte mentre delle figure androcefale gli aprono le porte della montagna, permettendo al Sole di sorgere (Matthiae 2000, 95; Margueron 2004, 419).

46 Parrot 1958a, 78; Margueron 2004, 418.

47 Parrot 1958a, 77; Matthiae 2000, 95; Margueron 2004, 418.

48 La stessa tipologia di tiara è indossata dal re Zimri-Lim nella famosa scena “d’investitura di Zimri-Lim" ritrovata nella Corte 106.

49 Esattamente come fa, nella scena del registro superiore, la dea intercedente che offre una libagione alle dea Ishtar assisa in trono.

50 Secondo Parrot si tratterebbe della dea paredra di Martu, Ashratu (Parrot 1958a, 78).

51 Questo fu uno degli aspetti chiave sottolineato da Moortgat nel giustificare la sua datazione dei dipinti della Stanza 132 al periodo neo-sumerico (Gates 1984, 77).

52 Questa figura ha avuto differenti interpretazioni: Parrot la definisce il dio Martu o forse Adad, mentre Margueron pensa sia il figlio di un re o un suo servo personale. Entrambi gli archeologi sono però concordi nel pensare ad una figura d'alto rango (Parrot 1958a, 78; Margueron 2004, 419). 
lungo contenente probabilmente una libagione; nell'altra mano regge una mazza, altra

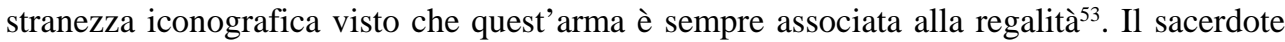
indossa una gonna bianca, una tiara bianca, che appare più schiacciata e con i bordi in maggior rilievo rispetto a quella del re, e porta una barba lunga e nera. Alle spalle del sacerdote vi è un vaso zampillante, simbolo di fertilità; segue una figura peculiare ${ }^{54}$ senza paragoni iconografici ad oggi ${ }^{55}$, disegnata su uno sfondo giallo nell'atto di reggere o tenere la volta celeste ${ }^{56}$. Il personaggio ha la pelle colorata di nero ed è l'unica figura, tra quelle presenti in tutti i registri, che ha i contorni dipinti di rosso. Lo stesso colore è impiegato anche per la volta celeste, che ha uno sfondo nero picchettato con dei tondini bianchi.

La lettura che si propone di questo registro della Stanza 132 acquista un significato se si associa il contesto, qui dipinto, con il rituale di sostituzione del re prima descritto. Sicché, procedendo nuovamente da sinistra, troviamo un toro passante, simbolo solare ${ }^{57}$, che si muove sulla montagna in direzione opposta al dio Sin. Questo gesto, letto in chiave cosmologica, potrebbe avere il senso del passaggio del Sole alle spalle della Luna, come avviene durante un'eclissi solare. Subito dopo troviamo il dio Sin stranamente assiso su una montagna, posizione in cui sarebbe logico trovare il dio Shamash ${ }^{58}$. Interpretando la figurazione da un punto di vista cosmologico, appare evidente che, essendo rappresentata una eclissi di Sole, il dio-Luna Sin sieda al posto del dio-Sole Shamash "usurpandone" momentaneamente il posto. Giungiamo alla figura del re che offre una libagione al dio Sin. In questo caso a versare la libagione non sarebbe altri che il re "fantoccio", che si appresta ad essere sacrificato; il re sostituto indossa, come lo stesso rituale attesta ${ }^{59}$, la tiara e la veste a balze di lana. Inoltre il sostituto è più basso delle altre figure rappresentate nella scena, denotando in questo modo che il suo ruolo è solo rituale e non reale; egli non è il vero re, ciò viene brillantemente evidenziato con alcune "stonature" iconografiche. Altro indizio a

53 Zimri-Lim nella scena d'investitura riceve dalla dea Ishtar una barra ed una fune, esattamente come Hammurabi nella celebre stele. Qui i simboli della regalità cambiano rispetto a quelli della Stanza 132 a causa della differenza cronologica e stilistica dei dipinti. Nella scena d'investitura sono chiare le ispirazioni babilonesi e siriane, mentre nella rappresentazione della Stanza 132 appaiono evidenti gli influssi mesopotamici, più vicini alla stele di Ur-Nammu. Lo stesso Margueron, parlando di questo sacerdote, si chiede se sia uno dei figli del re o un servitore, questa deduzione è dovuta alla mazza, simbolo regale. Margueron precisa che forse questa figura veste un ruolo di sacrificatore, vista la mazza e la libagione che porta, del toro rappresentato sul monte (Margueron 2004, 419).

54 Parrot sostiene che possa trattarsi del dio-Sole Shamash nell'atto di sorge al mattino annullando le tenebre (Parrot 1958a, 81).

55 Margueron 2004, 419

56 Secondo Matthiae questa figurazione ricorda l'immagine degli Atlanti di epoca protodinastica (Matthiae 2000, 96).

57 Il toro viene spesso associato al dio Shamash in diversi contesti. Nell'epopea di Gilgamesh, ad esempio, il dio Shamash appare in sogno a Gilgamesh sotto forma di toro (tavola IV detta "tavola dei sogni", il sogno è il quinto); inoltre sono sempre delle figure androcefale a spalancare le porte della montagna sacra e a permettere il sorgere del Sole.

58 Vedi nota 46; Matthiae 2000, 95; Margueron 2004, 419.

59 Come già detto, il re sostituto deve indossare i simboli della regalità per garantire l'efficacia del rituale e darne un senso allo scopo; infatti la finalità magica del rito è quella di far avverare realmente la predizione negativa che l'eclissi ha portato. Così che, sacrificando un sostituto, si possa salvare la vita del vero re (Frankfort 1978, 263). 
favore di questa interpretazione è l'atteggiamento della mano destra, che dovrebbe impugnare la mazza, accostata al fianco sinistro. Il sacerdote che porta il vaso per libagioni e la mazza non sarebbe altri che il vero re; a supporto di questa tesi si deve notare, oltre la detenzione della mazza, anche l'altezza. Questo personaggio è infatti il più alto tra le figure poste in piedi60 , l'unico a "toccare" con la testa la fascia decorativa che divide i due registri maggiori; anche il re sostituto effettivamente è posto alla stessa altezza del vero re, ma solo perché si trova sul gradino creato dalla montagna. Senza questo gradino, la figura del re sostituto risulterebbe la più bassa all'interno del registro. Infine, vi è il personaggio colorato in nero, l'ultimo a destra, che potrebbe essere una rappresentazione, forse sotto forma di divinità, di un'eclissi solare di tipo anulare. Si spiegherebbe, in questo modo, innanzi tutto la particolare scelta cromatica utilizzata per questa figura; se, infatti, si osservano i colori tipici di un'eclissi solare si trovano delle chiare corrispondenze con quelli usati per questo personaggio:

- il rosso utilizzato per i contorni ricorderebbe il tipico anello rosso che si forma per la sovrapposizione tra la Luna ed il Sole durante un'eclissi di tipo anulare; sicché il personaggio dipinto sarebbe il "nuovo" astro, o divinità, che viene a crearsi quando la Luna ed il Sole si incontrano;

- attorno a questa figura, tra il suo corpo e la volta stellata, vi è un campo colorato di giallo, lo stesso colore utilizzato per lo sfondo della scena. Questa fascia gialla sarebbe la luce emanata dal Sole, coperto, nelle sue immediate prossimità;

- la volta stellata non sarebbe altro che l'immagine simbolica del giorno che si fa notte, le stelle visibili in pieno mattino.

Tutto il registro non sarebbe altro che un istante congelato nel tempo: il re sostituto che versa le libagioni al dio-Luna Sin assiso sul trono di Shamash (sostituendolo e spodestandolo momentaneamente, come avviene nell'eclissi), mentre alle sue spalle il vero re di Mari, con un'altra libagione da versare, è pronto con la mazza a sacrificare la vita del sostituto nel culmine della cerimonia. In un riquadro separato e con colori diversi, si staglia la rappresentazione visiva del fenomeno dell'eclissi solare, che ha condotto la corte all'applicazione del rito di sostituzione; si potrebbe ipotizzare una rappresentazione di un dio-eclissi ${ }^{61}$, e, se dovesse davvero essere così, questa ne sarebbe l'unica attestazione ad oggi esistente.

\section{CONCLUSIONI}

Incertezze si sono sempre avute anche riguardo al significato delle pitture poste nella parete ovest della Stanza 132; nessun dubbio, ovviamente, circa il senso religioso e sacro dei registri maggiori, ma, al contempo, le principali interpretazioni condotte da Parrot sono molto esplicite e dirette, ed alcune anche possibili. Non vi è, però, nell'analisi portata avanti

60 È più alto sia del re sostituto, sia della dea intercedente che lo precede (fig. 1).

61 In questo registro è rappresentato un rito, o comunque una scena sacra appare chiaro che siamo in un contesto altamente simbolico in cui difficilmente si può scorgere un tentativo di rappresentare la mera realtà. Ciò deve essere avvenuto anche per la figura del dio-eclissi, che è tale perché sarebbe fuori luogo ipotizzare una semplice rappresentazione figurata di un fenomeno celeste senza che esso abbia un significato sacro, specie in un contesto simile. 
da Parrot, nessuna unità d'insieme: se la descrizione di ogni figura potrebbe essere plausibile, allo stesso tempo la sintesi d'insieme della raffigurazione è totalmente assente. Margueron dal canto suo, persino negli scritti più recenti, pare restio a voler dare una lettura critica del registro. Nonostante ciò, la sua analisi è quella che più converge, sebbene solo in alcuni punti, con quella riportata in questa tesi. Il pregio di collegare la scena rappresentata con il rituale di sostituzione è sicuramente quello di riuscire a dare un senso generale al registro: ogni soggetto recita una precisa parte che è a sua volta connessa con la presenza degli altri "attori"; inoltre, grazie a questa lettura, anche lo strano personaggio dipinto di nero nel registro, separato a sinistra, acquista un significato. Bisogna precisare che la mancanza di paragoni possibili con altre opere (visto che si tratterebbe, al momento, dell'unica rappresentazione visiva del rituale di sostituzione) relega solo ad una ipotesi questa lettura critica. Pesa, inoltre, la mancanza di notizie ulteriori e più dettagliate circa il rituale di sostituzione nel XX-XIX secolo.

Queste lacune non rendono comunque meno solida l'ipotesi di partenza che può essere corroborata da un interessante database on-line ospitato dal sito della NASA ${ }^{62}$; il progetto è coordinato da Fred Espenak, un astrofisico americano che ha concentrato molti suoi sforzi nella creazione di un database che includesse le eclissi solari e lunari per il periodo 3000 a.C. - 3000 d.C. Scorrendo l'archivio elettronico, si possono notare due eclissi solari visibili a Tell Hariri ed avvenute il: 24 giugno del 1916 a.C.63, di tipo anulare; 24 giugno 1832 a.C., di tipo totale ${ }^{64}$. L'esistenza di due eclissi solari, che coincidono ${ }^{65}$ con il periodo preso in considerazione per la realizzazione delle pitture parietali, potrebbe essere un ulteriore indizio a favore della connessione tra la scena rappresentata, il sostituto reale e le eclissi realmente avvenute e visibili a Mari. Questo dato chiaramente non ha il peso di un'attenta e scrupolosa analisi stilistica ed iconografica dell'opera, ma certamente è un ottimo indizio circa la possibile veridicità di quanto qui sostenuto. Sarebbe d'uopo sperare che possa essere ritrovato qualche altro esempio, meglio documentato o più certamente associabile, di rappresentazione figurata del rituale di sostituzione. Lo stesso ci si augura in relazione all'ottenimento di maggiori informazioni circa il rituale. Questi ed altri elementi ancora fanno pesare la loro assenza, e non permettono a questa tesi di uscire dal labirinto delle ipotesi.

62 http://eclipse.gsfc.nasa.gov/eclipse.html.

63 Questa eclissi, del tipo anulare, coincide con la possibilità vagliata da Margueron che il dipinto possa essere datato al XX secolo più che al XIX. Inoltre è da sottolineare che è proprio un'eclissi solare di tipo anulare quella che meglio sposa la tesi qui sostenuta (Margueron 2004, 416).

64 Come accennato precedentemente Matthiae, e con qualche riserva anche Margueron, reputano possibile che il dipinto sia stato eseguito nel XIX secolo (Matthiae 2000, 95; Margueron 2004, 416).

65 È rimarchevole il fatto che un'eclissi solare è visibile solo in alcune fasce della terra per volta, e che ciò avvenga abbastanza di rado. Non si tratta quindi né di un fenomeno che, quando avviene, ha una visibilità ampia; né di una coincidenza astrale che temporalmente si ripete spesso, in particolare le eclissi totali o anulari che sono quelle che conducevano più spesso al rituale di sostituzione. 


\section{BIBLIOGRAFIA}

BOTTÉRO, J.

1991 Mesopotamia. La scrittura, la mentalità e gli dei, Torino 1991.

BRELICH, A.

$1991 \quad$ Introduzione alla storia delle religioni, Roma 1991.

DURAND, J.M.

1995 Mitología y Religión del Oriente Antiguo II/1: semitas occidentales (Ebla, Mari) (Colección: Estudios Orientales 8), Sabadell 1995.

2000 Les documents épistolaires du palais de Mari, tome III (Littératures anciennes du ProcheOrient 18), Paris 2000.

FRANKFORT, $\mathrm{H}$.

1978 Kingship and the Gods: a study of ancient near eastern religion as the integration of society and nature, Chicago 1978.

GATES, H.M.

1984 The palace of Zimri-Lim at Mari: Biblical Archaeologist 47/2 (1984), pp. 70-87.

HUBERT, H. - MAUSS, M.

1981 Sacrifice: it’s nature and function, Chicago 1981.

KoCH-WeSTENHOLZ, U. - Koch, U.S.

1995 Mesopotamian astrology: an introduction to babylonian and assyrian celestial divination, Copenhagen 1995.

MARGUERON, J.C.

1982 Recherches sur les palais Mésopotamiens de l'age du Bronze. Tome 1 texte, Paris 1982.

2004 Mari. Métropole de l'Euphrate au III et au début du II millénaire av. J.C., Paris 2004.

MATTHiAe, $P$.

2000 La storia dell'arte dell'Oriente Antico: gli stati territoriali (La storia dell'arte dell'Oriente antico 2), Roma 2000.

PARPOLA, S.

2007 Letters from assyrian scholars to the kings Esarhaddon and Assurbanipal: part II commentary and appendices, Winona Lake 2007.

PARROT, A.

1958a Mission Archéologique de Mari. Volume II. Le Palais: peintures murales, Paris 1958.

1958b Mission Archéologique de Mari. Volume II. Le Palais: architecture, Paris 1958.

1974 Mari. Capitale fabuleuse, Paris 1974. 


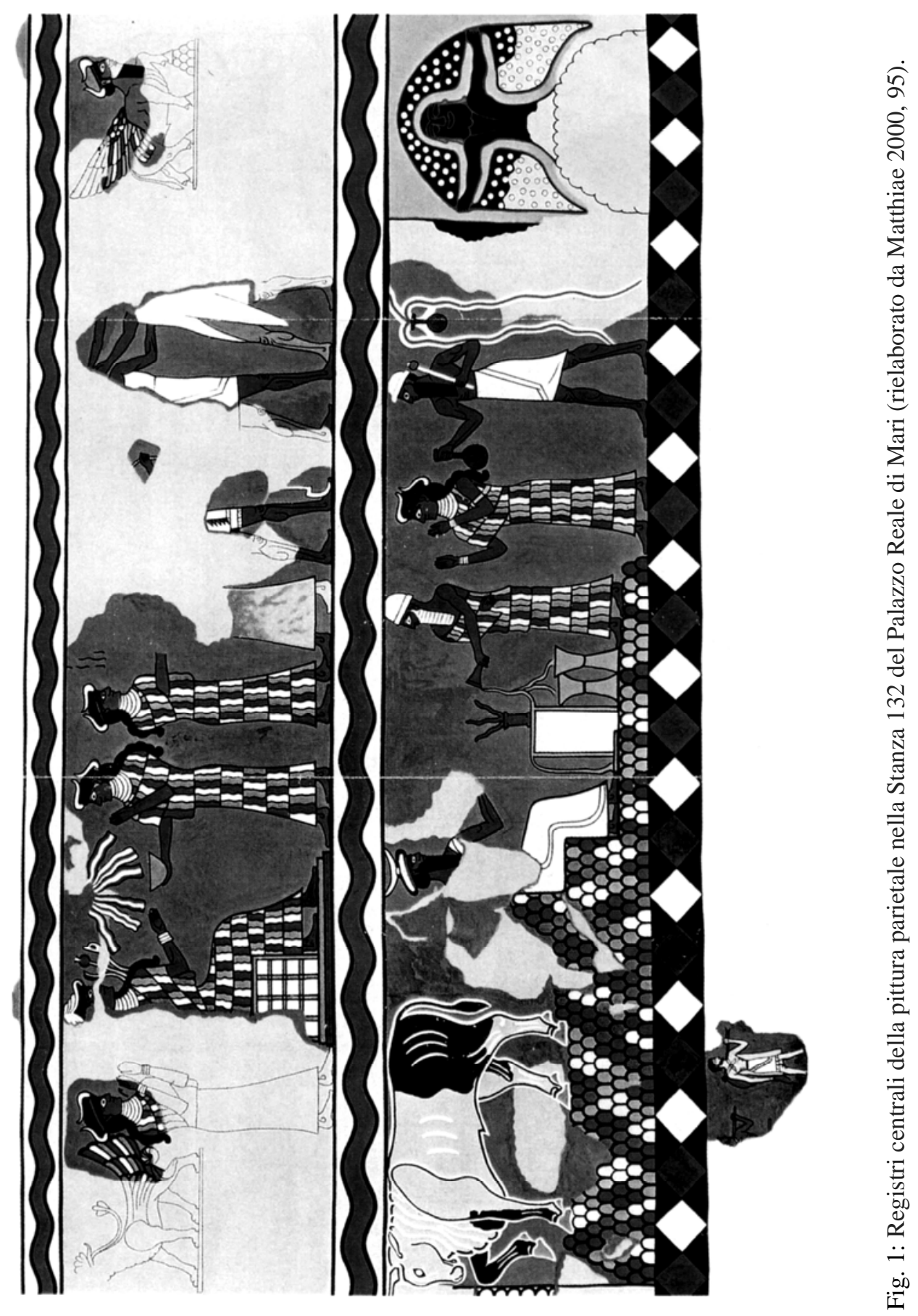

